\title{
SOMMEIL DU JUSTE ET DU SCÉLÉRAT : LES USAGES NARRATIFS DU RÊVE DANS UN ROMAN FLEUVE CHINOIS DU XVII ${ }^{\mathrm{E}}$ SIËCLE
} Vincent Durand-Dastès

\author{
Presses universitaires de Vincennes | « Extrême-Orient Extrême-Occident 》
}

2018/1 n 42 | pages 153 à 178

ISSN 0754-5010

Article disponible en ligne à l'adresse :

https://www.cairn.info/revue-extreme-orient-extreme-occident-2018-1-page-153.htm

Distribution électronique Cairn.info pour Presses universitaires de Vincennes.

(C) Presses universitaires de Vincennes. Tous droits réservés pour tous pays.

La reproduction ou représentation de cet article, notamment par photocopie, n'est autorisée que dans les limites des conditions générales d'utilisation du site ou, le cas échéant, des conditions générales de la licence souscrite par votre établissement. Toute autre reproduction ou représentation, en tout ou partie, sous quelque forme et de quelque manière que ce soit, est interdite sauf accord préalable et écrit de l'éditeur, en dehors des cas prévus par la législation en vigueur en France. Il est précisé que son stockage dans une base de données est également interdit. 
Sommeil du juste et du scélérat : les usages narratifs du rêve dans un roman fleuve chinois du $\mathrm{XVII}{ }^{\mathrm{e}}$ siècle

The Sleep of Heroes and Villains: Narrative Functions of Dreams in a 17th

Century Full-length Novel

安眠與驚寐: 夢在一部十七世紀長篇小説中的敘事功能

\section{Vincent Durand-Dastès}

\section{OpenEdition}

Journals

Édition électronique

URL : http://journals.openedition.org/extremeorient/969

DOI : 10.4000/extremeorient.969

ISBN : 978-2-84292-990-9

ISSN : 2108-7105

Éditeur

Presses universitaires de Vincennes

Édition imprimée

Date de publication : 1 décembre 2018

Pagination : 153-178

ISBN : 978-2-84292-988-6

ISSN : 0754-5010

Distribution électronique Cairn

\section{CAIRN INFO}

CHERCHER, REPÉRER, AVANCER

\section{Référence électronique}

Vincent Durand-Dastès, "Sommeil du juste et du scélérat : les usages narratifs du rêve dans un roman fleuve chinois du XVII siècle», Extrême-Orient Extrême-Occident [En ligne], 42 | 2018, mis en ligne le 01 décembre 2020, consulté le 04 mars 2019. URL : http://journals.openedition.org/ extremeorient/969; DOI : 10.4000/extremeorient.969 


\title{
Sommeil du juste et du scélérat : les usages narratifs du rêve dans un roman fleuve chinois du XVII ${ }^{e}$ siècle
}

\author{
Vincent Durand-Dastès
}

Dans cette courte étude, nous avons choisi de nous concentrer sur l'usage du récit de rêve dans un roman du XVII ${ }^{\mathrm{e}}$ siècle. Le choix de la période est loin d'être ici arbitraire : les traces d'un grand intérêt pour le rêve sont en effet nombreuses à la fin des Ming et au début des Qing. On en relève non seulement dans les œuvres de fiction, les poèmes ou les «notes au fil du pinceau », mais également au travers de la publication de volumineux traités d'onirologie comme le Mengzhan yizhi (1562) de Chen Shiyuan (1516-1595) et d'autres sommes encyclopédiques ${ }^{1}$. On pourrait aussi évoquer l'activité d'un auteur comme Dong Yue (1620-1686) qui, outre son choix du cadre onirique pour son roman le Xiyou bu, était connu pour avoir noté ses propres rêves et partagé ses expériences oniriques avec d'autres lettrés ; il fut loin d'être un cas isolé ${ }^{2}$. Bref, bien des indices montrent l'existence d'un haut degré d'ouverture au rêve dans la société de cette époque.

\section{Chan zhen yishi, Les Histoires oubliées des maîtres de dhyāna et des parfaits}

La fin des Ming est aussi ce que l'on pourrait qualifier d'âge d'or du roman en langue vulgaire tongsu xiaoshuo. Au fil des pages des récits, courts

1. Sur les traités onirologiques Ming, voir notamment Lackner 1985, Drettas 2008, Vance 2012, ainsi que l'article de cette dernière dans ce numéro. Voir aussi Liu Wenying 2003.

2. Le Хiуou $b u$ fut achevé en 1641 , encore pendant la première moitié du XVII ${ }^{\mathrm{e}}$ siècle. Hegel 1981 : 162-163 ; Zeitlin 1993 : 136. Sur l'importance des rêves dans les écrits autobiographiques d'un contemporain de Dong, Xue Cai (1598-1665), voir Struve 2007. 
ou longs, qui constituent ce genre, le lecteur ne peut manquer d'être frappé par l'abondance quantitative, mais aussi par ce qu'on aurait envie d'appeler la haute valeur qualitative, des épisodes oniriques qu'on y découvre. Nous avons choisi d'explorer systématiquement ici les épisodes oniriques du roman Chan zhen yishi, dont le traitement des séquences rêvées nous a paru tout à fait remarquable. En nous attachant à un récit singulier dans son entier, nous espérons en outre pouvoir mettre en lumière les différents usages narratifs et rhétoriques par lesquels une même œuvre peut investir le récit onirique.

Le roman intitulé « Histoires oubliées des maîtres de dhyāna et des parfaits taoïstes » Chan zhen yishi, en 40 chapitres, fut composé pendant les premières décennies du XVII ${ }^{\mathrm{e}}$ siècle ${ }^{3}$. Il est signé du nom de pinceau "Le taoïste du torrent pur », Qingxi daoren, qu'on estime avoir été utilisé par un habitant de Hangzhou du nom de Fang Ruhao, sur qui on ne sait par ailleurs presque rien ${ }^{4}$. Il fut prolongé par un second roman, « Histoire postérieure des maîtres de dhyāna et des parfaits »Chan zhen houshi, en 60 chapitres, également par Fang ${ }^{5}$, ce qui constitue un des rares exemples d'un roman initial et de sa suite à avoir été rédigés par un même auteur : nous ferons aussi appel dans une moindre mesure à ce second texte. Bien que ces deux romans aient été assez souvent réédités, de l'époque impériale à nos jours, ce qui témoigne d'une certaine popularité ${ }^{6}$, ils ont longtemps été négligés par la critique, les premières

3. Sur la date de parution de Chan zhen yishi, généralement estimée avoir eu lieu sous l'ère Tianqi (1620-1627), voir Durand-Dastès 2008 : 268 note 607. Voir aussi Song Yan, pour qui le roman a dû être publié au plus tard en 1626 : Song Yan 2014-5 : 9-11.

4. Sur le nom de pinceau Qingxi daoren et son identification à Fang Ruhao, voir DurandDastès 2008 : 266-284 et Jia Haijian 2013 : 199-209.

5. Chan zhen houshi porte une préface de 1629. Durand-Dastès 2008 : 268 note 607. Song Yan 2014-5 : 9-11.

6. Il est toujours difficile d'évaluer la diffusion d'un roman chinois de l'époque impériale tardive. Le fait qu'il ait été plusieurs fois réédité, d'une part, et qu'un nombre significatif d'exemplaires de ces éditions anciennes soient parvenu jusqu'à nous, d'autre part, permet d'attester qu'un roman a suscité un intérêt relativement constant. Dans le cas de Chan zhen yishi, neuf maisons d'éditions différentes publièrent le roman (Wang Qingyuan et al. 2002 : 293, 302, 321) entre la fin des Ming et la fin des Qing. Sous l'ère Guangxu (1875-1908), un même éditeur, la Shanghai shuju, n'en produisit pas moins de trois éditions lithographiques, sous trois titres différents : Chan zhen yishi, Canliang wai shi «Histoire non officielle de la perte des Liang » et Miaoxiang si quanzhuan " Histoire complète du monastère Miaoxiang ». Une édition du roman portant ce dernier titre fut encore réalisée à l'époque républicaine (Tarumoto 2002 : 481). Les éditions de Chan zhen houshi sont moins nombreuses : il en subsiste quatre, dont une datée de 1629 , probablement la première édition, plus une édition expurgée des sept chapitres les plus osés. Si les éditions Ming des deux romans sont des unica, 
études les concernant, qui restent peu nombreuses, n'ayant paru qu'à partir des années 1980.

Fang Ruhao est remarquable, comme l'a noté un de ses premiers commentateurs, Keith McMahon, par son aptitude à reproduire dans son œuvre presque tous les styles et genres du roman en langue vulgaire de son temps, depuis le roman de mœurs jusqu'à la pornographie en passant par les roman de guerres magiques, le roman historique ou les récits de preux chevaliers ${ }^{7}$. Vu de notre $\mathrm{XXI}^{\mathrm{e}}$ siècle, Fang apparaît comme un personnage assez peu sympathique : dans ses récits, les déviants et les marginaux sont impitoyablement combattus et châtiés, parfois avec cruauté ${ }^{8}$. Mais, grâce à ses brillants talents de styliste, il nous fait aussi entendre d'émouvante façon la voix même de ceux qu'il condamne $^{9}$, et la lecture de ses romans réserve d'excellents moments au lecteur d'aujourd'hui. Parmi ceux-ci, on peut sans hésiter ranger les descriptions de rêves, nombreuses dans les pages qu'il nous a laissées.

Nous allons donc emprunter à cet auteur tous les exemples retenus pour cet article. Leur originalité n'est cependant pas constante, et il conviendra de distinguer parmi les extraits ici retenus procédés rhétoriques et usages du rêve communs aux auteurs de sa génération des traits appartenant sans doute davantage en propre à notre romancier.

Parmi les premiers, nous pouvons citer les procédés rhétoriques grâce auxquels un rêve prend forme et s'achève dans un récit en langue vulgaire. Ici, Fang reprend très largement des techniques qu'il n'a pas inventées. Mais il les emploie avec une habileté et une efficacité saisissantes. Ces procédés habiles constituent aussi, me semble-t-il, de beaux exemples de ce qu'on pourrait appeler le «naturalisme onirique » qui fait l'intérêt et la beauté du récit de rêve tel qu'on peut le rencontrer dans maints romans en langue vulgaire.

on trouve plusieurs exemplaires des éditions Qing dans des collections publiques en Chine, au Japon, en Corée et en Europe (British museum, Université de Leyde). Pour une liste des éditions et des exemplaires transmis, voir Wang 2002 ; voir aussi Ōtsuka 1987 : 164-165 [numéros 23005 et 23006] ; Shi Lei 2004 : 25-26.

7. Mc Mahon 1988: 107.

8. Voir à ce sujet Durand-Dastès $2007: 86-100$.

9. McMahon $1988: 114-116$. 


\section{Entrer en songe, s'éveiller d'un cauchemar : les procédés d'inser- tion de l'épisode onirique dans le cours du récit}

Sauf bien sûr dans le cas où il est explicitement identifié comme tel par un personnage qui vient le narrer après-coup, un des enjeux du récit de rêve romanesque est de placer le lecteur dans la même inconscience de son état que le personnage endormi. Fang Ruhao s'y essaye avec bonheur, notamment en s'interdisant d'employer le mot « rêve » avant que celui-ci ne soit arrivé à son terme ${ }^{10}$. Ainsi, au commencement du rêve, on peut avoir dit explicitement que le personnage s'est endormi - et l'irruption du rêve est alors représentée comme son réveil, ou bien avoir simplement mentionné que la fatigue ou une certaine confusion s'est emparée du héros - et le surgissement du rêve est simplement marqué par l'intervention d'un nouveau protagoniste. Voici un exemple du premier cas :

Ayant passé la moitié de la nuit en supputations, il finit par se sentir mentalement épuisé, et se laissa tomber endormi, tout habillé. Soudain, il entendit quelqu'un frapper à la porte, et, prêtant l'oreille, reconnut la voix de son beau-frère Badang ; il se hâta d'ouvrir la porte pour le laisser entrer ${ }^{11}$.

Et un exemple du second :

10. Affirmer que le XVII ${ }^{\mathrm{e}}$ siècle chinois serait ici « en avance » sur l'Occident dans sa façon d'élider sciemment du récit le moment de l'entrée en rêve peut paraître hasardeux, mais certains indices vont dans ce sens. Dans le sous-chapitre « Frontières » de son étude sur le récit de rêve dans le roman européen moderne, Jean-Daniel Gollut rappelle ainsi que la littérature européenne du Moyen Âge marquait toujours explicitement le moment d'entrée et de sortie du rêve (Gollut 1993 : 64). S'il donne ensuite maints exemples de procédés par lesquels le début du rêve se voit élider dans le roman européen, la plupart de ceux-ci proviennent d'œuvres des XIX et $\mathrm{XX}^{\mathrm{e}}$ siècles. L'exemple le plus ancien d'une ellipse d'un début de rêve qu'il donne est toutefois emprunté à un conte de Voltaire, « Le crocheteur borgne » (Gollut $1993:$ 70).

11. 算計了半夜, 漸覺精神疲倦, 和衣睡倒。忽聞有人叨門, 側耳聽時, 乃是姐 夫巴富聲音, 慌忙開門迎入。(Chan zhen yishi, chapitre 12. Qingxi daoren 1986 : 177.) Je donne la pagination des références pour Chan Zhen yishi dans l'édition de 1986 de Heilongjiang renmin, qui a l'avantage de ne pas être censurée comme le sont beaucoup d'autres éditions modernes. On trouvera une reproduction photographique de l'édition Ming du livre en trois volumes dans la série Guben xiaoshuo jicheng, Shanghai guji, 1990. Des versions en ligne sont également facilement accessibles sur le site Kaifang wenxue (openlit.org) ou le site Ctext.org. 
Les usages narratifs du rêve dans un roman fleuve chinois du XVII ${ }^{\mathrm{e}}$ siècle

Il se tourna et se retourna sur lui-même à maintes reprises sans pouvoir s'endormir. L'approche de l'aube le trouva dans un grand état d'épuisement mental, et, l'esprit dans le vague, il s'appuya sur une balustrade près d'une pierre du lac Tai, regardant les poissons rouges s'ébattre dans la mare. Alors qu'il les regardait, un serviteur laïc vint lui annoncer : « il y a dans la Grand-salle du Bouddha une dame bodhisattva venue sceller un vœu par une récitation de sūtra : elle insiste, Monsieur l'abbé, pour que vous conduisiez en personne sa confession ${ }^{12}$. »

Un terme très fréquemment rencontré pour introduire le rêve sans dire explicitement que le héros s'endort est celui de menglong, qui peut signifier la pénombre ou une faible lueur, et désigner ce qui paraît voilé, flou, confus ou vague. On le rencontre souvent dans les descriptions d'entrée en rêve, comme celle-ci, qui évite grâce à lui l'emploi du mot « sommeil » :

Dans le vague, il ferma les yeux, et se sentit en train de porter un sceau, se dirigeant vers la rivière pour aller y puiser de l'eau ${ }^{13}$.

Par contraste, la sortie du rêve, pour être bien marquée, emploie en général explicitement le mot d'éveil (xing) sinon de rêve (meng) ou de cauchemar (yan), appuyant souvent la prise de conscience rétrospective du rêveur par l'expression proverbiale "C'était un rêve de Nanke ! » (Nanke yi meng) ${ }^{14}$. C'est cette exclamation qui viendra aux lèvres de l'abbé débauché Zhong Shoujing au sortir d'un rêve agité :

Zhong Shoujing se débattant de toutes ses forces sans parvenir à se dégager, s'écria : «Le dhüta ${ }^{15}$ m'assassine, à moi la milice ! » Le novice Laizhen, l'entendant crier ainsi, supposa qu'il faisait un cauchemar et se hâta de l'éveiller. Reprenant conscience, Zhong Shoujing comprit que ce n'avait été qu'un rêve de Nanke ; il

12. 翻來覆去, 再三睡不著。直捱到五更, 神思困倦, 朦朧在太湖石畔 , 憑著欄 杆看池裏金魚遊戲。正看間, 道人來 報: 「佛殿上一位女菩薩來許經願, 要 接住持爺親自懺悔。」(Chan zhen yishi, chapitre 5. Qingxi daoren 1986 : 63).

13. 朦朧合眼去, 覺自己挑了一副水桶, 往溪邊波水。(Chan zhen yishi, chapitre 12 . Qingxi daoren, 1986: 177).

14. Allusion au célèbre conte de Li Gongzuo ( ca 770-850), «La Biographie du gouverneur de Nanke » (Nanke taishou zhuan), dont le protagoniste croit traverser de longues années de sa vie le temps d'un simple songe.

15. Le terme de $d h \bar{u} t a$, qui désigne originellement un type d'ascètes ermites, sert souvent à désigner à l'époque Ming des moines errants peu soucieux des règles monastiques. Nous revenons plus loin sur le sens de cet épisode. 


\section{Vincent Durand-Dastès}

était couvert de sueurs froides, haletant sans pouvoir reprendre haleine, et ne faisait que soupirer en son for intérieur ${ }^{16}$.

Un peu plus loin dans le récit, ce n'est pas un réveil agité, mais deux, que le misérable traître Abao devra successivement endurer au cours d'une même nuit de trouble. Le premier :

Abao voulut crier qu'on lui vienne en aide, mais voilà qu'il se retrouva muet : luttant de toutes ses forces sans retrouver la voix dans son cauchemar, il fut entendu par le voisin mitoyen qui l'éveilla. Abao s'exclama à plusieurs reprise « pouah, pouah, pouah! » Son cœur battait à tout rompre, et il avait eu si peur qu'il était couvert d'une sueur glacée ${ }^{17}$.

et le second :

L'émissaire au visage rubicond souleva Abao et le projeta contre le mur voisin. Abao poussa un grand hurlement et s'éveilla soudain : il faisait déjà grand jour. En repassant en détail dans son esprit les circonstances de son rêve, il se sentit oppressé et mal à l'aise ${ }^{18}$.

Au-delà des moments charnières de l'entrée ou de la sortie du rêve, le récit de rêve montre souvent aussi une grande fidélité à l'aspect étrange, fluide et changeant de l'expérience onirique ${ }^{19}$. Ainsi, dans la dernière partie d'un rêve fait par un personnage de la seconde époque : la rêveuse est ici Lao Woxi, qui

16. 鍾守淨極力掙扎不得, 大聲喊叫：「頭陀殺人, 地方救命！」行童來真聽得喊 叫, 諒是鍾守淨夢魘, 慌忙叫喚。鍾守淨醒來, 卻是南柯一夢, 掙得一身冷 汗, 喘息不定, 心下暗暗嗟吁不已。(Chan zhen yishi chapitre 5. Qingxi daoren 1986 : 64).

17. 阿保喊叫救命, 奈何聲啞, 極力掙不出聲, 魘將起來。幸隔房聽得, 叫他 方醒。阿保連聲啐道：「吥, 吥, 吥！」心頭元自躑躑的跳, 驚得一身冷 汗。(Chan zhen yishi chapitre 12. Qingxi daoren 1986: 177).

18. 那赤臉使者, 將阿保提起來隔牆一撩, 阿保大叫一聲, 忽然驚覺, 天已大曉。 暗詳夢中境界, 悶悶不樂。(Chan zhen yishi chapitre 13. Qingxi daoren 1986 ： 179).

19. Gollut écrit joliment à propos de l'irruption de l'insolite lorsqu'un récit devient récit de rêve, même sans avoir marqué le commencement de celui-ci : «le rêve "clandestin" se trahit quelque peu - avant de se découvrir franchement - par le caractère insolite du monde représenté. L'attention du lecteur est ainsi plus ou moins alertée et la mise au point finale (du genre : «ce n'était qu'un rêve ! ») fait somme toute office de solution au problème d'intelligibilité auquel a pu se sentir confronté jusque-là le destinataire du récit » (Gollut 1993 : 77). 
a été appelée par son époux pour rejoindre l'épouse principale et les autres concubines pour boire en contemplant les fleurs. Mais le décor idyllique va soudain devenir bien plus inquiétant :

Soudain une grande flamme ayant la taille d'une roue descendit en volant du nord-ouest, et se mit à tourbillonner à plusieurs reprises, enflammant tout autour du pavillon. Tous ceux qui s'y trouvaient se dispersèrent en grand tumulte. Woxi, affolée, voulait s'enfuir, mais ses deux jambes étaient comme entravées par des liens et elle ne pouvait bouger. Le cœur battant et tremblant de terreur, elle se mit à appeler au secours en hurlant. Mais voilà que le moine laissa tomber son tambour de bois, et, levant les deux larges manches de sa robe de bonze, se mit à repousser l'eau du fleuve pour l'en asperger à grandes brassées, si bien qu'en un instant elle eut de l'eau jusqu'à la taille. Woxi se mit à hurler : «Assez d'eau !!! » Mais le moine répondit : « Sans eau, comment ton feu voudra-t-il s'éteindre?» Et sans plus se soucier de ses protestations, il se mit à verser l'eau du fleuve. Woxi, voulant à tout prix échapper à l'inondation se mit à courir, mais une vague glacée la gifla, et frissonnante, elle fit un bond et s'éveilla de terreur : ce n'avait été qu'un rêve de Nanke ${ }^{20}$.

\section{Le maillage onirique du récit : de la simple ponctuation au tournant narratif}

Comme nous l'avons dit, Fang Ruhao est loin d'être le seul parmi ses contemporains à employer des procédés rhétoriques comme ceux que nous venons d'examiner. Un autre trait que l'on peut également dire partagé par Fang avec les auteurs de romans de son temps est la fréquence et la régularité

20. 忽然西北上飛下一塊火光, 大似車輪, 就地滾了幾遍, 焰騰騰把亭子四園燒 着。亭子內眾人一鬨而散。我惜驚惶，也欲奔走，奈兩腳似繩子絆住的一般不 能移動, 心慌膽顫, 高聲喊叫救人。只見那和尚粉下手中的梆子, 舉起兩支褊 衫大袖, 抄起江水呼呼地潑將上來, 頃刻間亭中之水已過腰胯。我惜大叫道: 不要水了。和尚應聲道：「不用水你那火怎麼肯熄？」不顧前後，把江水亂潑 將來。我惜拼命冒水而走, 忽然臉上衝着一陣冷水, 打了一個寒噤, 驚閃醒 來, 卻是南柯一夢。(Chan zhen houshi, chapitre 51. Fang Ruhao 1993 : 460). Je donnerai la pagination des références pour Chan zhen houshi dans l'édition ponctuée et collationnée par Wang Yimin pour la collection Mingdai xiaoshuo jikan, qui présente l'avantage d'être une édition non censurée de la version en soixante chapitres. Comme pour Chan zhen yishi, on trouvera une reproduction de l'édition Ming dans la série Guben xiaoshuo jicheng. Une bonne version en ligne est accessible sur le site Wikisource, qui propose les deux versions, en cinquante-trois et soixante chapitres. 
de l'insertion de brefs épisodes rêvés au fil du récit. La caractéristique de ces songes-là est que la plupart d'entre eux ne donnent pas lieu à un développement substantiel. Ils prennent place comme des sortes de ponctuations oniriques dans le cours du roman. Relevons les brefs usages des songes qui émaillent le récit de Chan zhen yishi : au chapitre 1, la mère d'un des héros (le futur abbé débauché Zhong Shoujing) rêve qu'un tigre entre dans sa maison juste avant de tomber enceinte. Au chapitre 11, le brigand d'honneur Miaolong voit annoncée par un rêve sa rencontre avec le bonze chevaleresque Lin Danran. Au chapitre 20, le défunt bonze Yongqing vient demander au même Lin Danran de régler pour lui une dette qui le condamne à de terribles souffrances dans l'autre monde. Sa dette apurée grâce à l'intervention de Lin, il apparaît de nouveau en rêve pour remercier son bienfaiteur. Enfin, au chapitre 31, l'un des disciples de Lin, Xue Ju, dit avoir vu en rêve un grand et imposant officier, qui, assis entre deux grands arbres à califourchon sur un homme, et tenant dans ses mains des baguettes d'achillée divinatoires, lui a rudement reproché d'avoir manqué à venger son père. L'ami à qui il raconte son rêve a tôt fait de le lui expliquer : si l'homme tenait des baguettes d'achillée, c'est parce que c'est grâce à elles que l'on choisit, lorsque l'on procède à la divination à l'aide du Livre des mutations, les lignes yao 爻composant les hexagrammes; si l'homme était assis entre deux arbres 木et avait sous lui un homme 一人, c'était pour lui livrer les autres éléments composant le caractère Fan樊 : 爻+木+木+一人. L'ennemi dont il faut tirer vengeance ne peut-être que le général Fan Wurui 樊武瑞, soupçonné d'avoir été impliqué dans la mort du père, ce que le rêve vient de confirmer ${ }^{21}$.

Là encore, tout ceci n'a rien d'extraordinaire, et de semblables ponctuations oniriques, qui usent du songe, dans la tradition onéiromantique, essentiellement comme un présage d'évènements à venir (mengzhao), pourraient être relevées dans à peu près n'importe quel roman de la fin des Ming ${ }^{22}$.

Chan zhen yishi se distingue toutefois en ce qu'il comporte aussi des récits de rêve beaucoup plus développés, s'étendant parfois sur plusieurs pages, et qui vont jouer dans le roman un véritable rôle structurel. Ces séquences oniriques ne sont pas seulement dépeintes par Fang Ruhao avec brio et un

21. Nous avons affaire ici à une explication par la méthode glyphomantique appelée chaizi. Bien que pouvant se pratiquer hors contexte onirique, cette méthode est très fréquemment utilisée pour l'interprétation de rêves. Sur la méthode du chaizi en général, voir Bauer 1979. Pour des exemples anciens d'emploi de la technique pour interpréter les rêves, voir Strassberg 2014. Sur le rôle de la méthode dans les traités oniromantiques Ming, voir Vance 2017.

22. Voir les exemples donnés dans Wan 2005 : 222-237. 
grand talent dans la restitution de la « chair» de l'expérience onirique : elles ont aussi pour fonction d'aider à structurer le récit et à articuler les épisodes entre eux. Les rêves ayant les échos les plus décisifs sur d'autres segments du récit, sont, on ne s'en étonnera pas, ceux qui touchent à Thanatos et à Eros. Dans le premier type de rêve, que nous nommerons rêve infernal ou « rêve yin » (yinmeng), le rêveur fait la rencontre d'un mort ou est lui-même entraîné aux enfers pour y être jugé ou admonesté avant d'être renvoyé dans le monde des vivants. Le second type, le « rêve printanier » (chunmeng), transpose dans l'espace du sommeil désirs et attentes sexuelles ou sentimentales du rêveur. Tout en lui permettant de les assouvir en partie, il lui laisse un troublant résidu de frustration ou d'inachèvement, résidu qui fournira la matière d'un nouveau développement du récit. Nous allons maintenant examiner plus en détails plusieurs séquences oniriques sophistiquées illustrant chacune un de ces types de rêves.

\section{Le rêve infernal de Chen Abao ou les cauchemars d'un méchant de basse extraction}

Les chapitres 12 et 13 de Chan zhen yishi nous offrent une intéressante double séquence onirique. Le rêveur se nomme Chen Abao, et il compte sans ambiguïté parmi les méchants de l'histoire. Abao est un tout jeune homme, gueux et sans scrupule, qui a trahi, pour toucher une récompense, Lin Danran, un des principaux héros du récit, et qui a entraîné, ce faisant, la mort d'un autre personnage positif, le noble et généreux Du Chengzhi. Juste avant qu'il ne commence à rêver, Abao se sent frustré : un plus malhonnête encore que lui est parvenu à s'arroger une partie de la récompense promise pour sa trahison et, bien qu'il soit resté en possession de cent taëls d'argent, son esprit ne parvient pas à trouver le repos : doit-il confier la garde du magot à son patron (qui risque bien de se servir au passage) ? Enterrer son trésor (mais ne pourraitil pas être observé et volé) ? Le garder constamment sur lui (dangereux et peu commode...) ? C'est dans cet état d'agitation et d'incertitude qu'il finit par s'assoupir, pour être aussitôt réveillé par son beau-frère, venu lui proposer d'investir son capital dans un voyage commercial outre-mer. Les deux parents embarquent ensemble, mais leur bateau est bientôt pris dans une tempête et chavire. Abao parvient à avoir la vie sauve en s'accrochant à une planche à la dérive, puis à gagner le rivage d'une île inconnue. À peine revenu sur la terre ferme, il est pris à partie par un homme de haute stature qui tente de le rançonner. Voulant échapper à son agresseur, il tombe dans une fosse à purin où, perdant pied et recouvert d'insectes immondes, il est sur le point 
de se noyer. Il se débat alors si fort dans son sommeil que son voisin mitoyen l'entend et le réveille, mettant un terme à ce premier cauchemar. Se retournant sur lui-même, Abao ne tarde pas à se rendormir : le rêve dans lequel il entre alors est de prime abord beaucoup plus plaisant : voyant des poissons s'ébattre dans une rivière aux eaux limpides, Abao se déshabille et plonge dans l'eau à leur poursuite ${ }^{23}$. Mais voilà que le songe tourne de nouveau au cauchemar : Abao se voit soudain tiré brutalement hors de l'eau et jeté sur le pont d'un navire d'apparat où siège un haut mandarin, entouré de gardes à l'aspect démoniaque. Abao, toujours nu comme un vers, est ligoté et mis en accusation par le magistrat, qui fait apporter un registre où l'on peut lire qu'Abao est la réincarnation d'un boucher. Déjà promis en raison de ce mauvais karma à une brève existence de seulement trente-six années, il va voir son laps de vie encore réduit du fait de sa récente félonie. Le juge écrit sur le visage d'Abao un poème énigmatique disant : «Bonheur pour les justes, calamités pour les débauchés : le regard divin frappe comme la foudre. Le trésor reviendra aux deux printemps, les émoluments s'achèveront en soie blanche » (fushan huoyin, shenmu rudian; bao gui er chun, lu zhong yi lian), puis ordonne qu'on le chasse : un des démons-gardiens le saisit pour le projeter violemment vers un mur. Abao s'éveille de nouveau en sursaut, horrifié et haletant ${ }^{24}$.

\section{Le rêve printanier de Zhong Shoujing où comment le naïf abbé est initié à l'amour et à la frustration}

Le second rêve que nous allons examiner ici intervient plus tôt dans le récit de Chan zhen yishi, au chapitre cinq. Le rêveur est Zhong Shoujing, le jeune et influent abbé d'un grand monastère de Nankin à l'époque de la dynastie des Liang. Brillant sujet, devenu moine dans sa prime enfance, Zhong Shoujing est à présent un vrai moine mondain, voire un religieux à la mode, et côtoie ainsi grands personnages et jolies fidèles. Il n'est d'ailleurs pas sans commencer à déplorer les contraintes de l'état monacal. Déjà, au chapitre 4, des cambrioleurs,

23. Ce début rappelle fortement l'incipit du conte Tang Xue Wei, traduit par André Lévy sous le titre de "Carpe fut », et qui conte la métamorphose provisoire en poisson d'un homme tombé en catalepsie. Là aussi, la vision plaisante tourne au cauchemar, puisque le poisson est pêché et découpé pour le banquet funéraire commandé par les amis du héros....lequel se réveille alors en sursaut, revenant à la vie. Voir Lévy (trad.) 1998 : 167-175. Le conte Tang, qui fut adapté au format de la nouvelle en langue vulgaire au XVII ${ }^{\mathrm{e}}$ siècle, a pu inspirer Fang Ruhao.

24. Chan zhen yishi chapitre 12 et 13. Qingxi daoren $1986: 177-179$. 
attirés par les richesses que ce monastère patronné par la famille impériale ne devrait pas manquer de renfermer, ont pénétré nuitamment dans le monastère. Ils ont alors surpris Zhong Shoujing endormi en train de marmonner dans son rêve une phrase qui indique sans ambiguïté à leurs yeux qu'il est en train de faire un «rêve printanier », impliquant sans doute, pensent-ils, un de ses petits novices ${ }^{25}$. Le lecteur n'en saura pourtant pas davantage sur ce rêve, mais il anticipe en quelque sorte le songe du chapitre suivant qui, lui, sera livré avec tous ses détails par le narrateur.

Zhong est tombé amoureux au premier coup d'œil d'une jolie jeune femme venue ce jour-là assister à son prêche, mais il ignore tout d'elle et ne sait comment la rencontrer de nouveau. Incapable de chasser la belle de son esprit, il passe toute la nuit sans trouver le sommeil. À l'approche de l'aube, il est sorti prendre l'air dans son jardin quand un novice vient lui dire qu'une femme demande à lui parler en vue de passer commande d'une récitation de sūtra. Zhong est ravi de découvrir en la rencontrant celle-là même qui ne quitte plus son esprit. Elle lui offre deux taëls d'argent pour prix de la cérémonie, mais, lorsque Zhong Shoujing veut recevoir la somme de ses mains, il s'aperçoit qu'elle lui a tendu son épingle de tête. De plus en plus troublé, il lui demande son nom, mais la belle répond en récitant un poème, apparemment sans queue ni tête. Zhong l'interrogeant sur le motif de sa démarche, elle confesse, que, étant enceinte, elle veut s'assurer que son enfant soit de sexe mâle. Zhong, prétendant posséder un « cinabre miraculeux » (lingdan) propre à lui assurer la descendance masculine espérée, l'attire jusqu'à sa cellule. Parvenu en ce lieu, il lui réclame comme prix supplémentaire de ses services "le trésor vivant qu'elle porte au milieu de la taille, plus précieux que vingt mille taëls d'or pur »...Exhibant alors son pénis, il lui explique que le médecin sera « ce petit moine, aux soins vraiment miraculeux » et enlace la belle. Mais il est interrompu par l'irruption soudaine "d'un moine errant dhüta (toutuo) au visage écarlate surgi d'une fente de la muraille » (hu jian bifeng li zanchu yi ge honglian toutuo ), qui se met à le rouer de coups. Zhong appelle à l'aide en hurlant si fort qu'il attire l'attention d'un jeune disciple qui arrive près de lui... et l'éveille de ce qui n'était qu'un songe ${ }^{26}$.

25. Chan zhen yishi chapitre 4. Qingxi daoren 1986: 50 .

26. Chan zhen yishi chapitre 5. Qingxi daoren 1986:63-64. 


\section{Suites immédiates des rêves : vraies et fausses explications oniromantiques}

Les héros des deux rêves sortent troublés et affectés par leurs expériences oniriques. De façon tout à fait caractéristique, ils vont tous deux se mettre en quête d'une sorte d'analyste capable de leur en expliquer la signification. L'infortuné Chen Abao ira rechercher ces éclaircissements auprès d'un moine taoïste, un débauché de la pire espèce, qui, après s'être vu répéter la phrase écrite en rêve sur le visage d'Abao, lui donne pour explication sommaire que le mot « printemps » qu'elle contient signifie que son rêve était en dépit des apparences un rêve érotique printanier, un chunmeng ${ }^{27}$ : il mène Abao dans un bordel rencontrer une courtisane renommée du nom de Meichun («Printemps charmant »). Mais Abao se verra finalement en ces lieux humilié et dépouillé de tout son argent par Meichun : désespéré, il finira par se pendre ${ }^{28}$. Nul n'a su lui expliquer le véritable sens du poème du magistrat nautique : comme l'annonçait la phrase «Le trésor reviendra aux deux printemps » son argent était destiné à aller tout en entier à la courtisane justement nommée «Printemps » et à son amant Han Huichun (dont le prénom signifie « Retour du printemps ») ; la strophe disant «que les émoluments finiraient en soie blanche » faisait allusion au cordon de soie par lequel Abao devait finalement mettre un terme à son existence.

En revanche, la personne consultée par Zhong Shoujing, pas moins immorale que le taoïste débauché, est autrement compétente en matière d'interprétation des rêves : il s'agit d'une veuve maligne et roublarde s'étant mis à porter l'habit monacal pour faciliter ses manigances, et dont l'art consommé du verbe retors lui a valu le surnom de Zhao mizui, Zhao « Bouche-de-miel ${ }^{29}$. Au chapitre 6, elle se montre capable de déchiffrer, via la divination par recomposition ou décomposition des sinogrammes chaizi $^{30}$, le mystérieux poème déclamé par la belle du rêve : il donne simplement le nom de la femme, Li Saiyu, et celui du mari, de celle-ci, Shen Quan ${ }^{31}$. Comme Bouche-de-miel exerce habituellement

27. Chan zhen yishi chapitre 13. Qingxi daoren $1986: 180$.

28. Chan zhen yishi chapitre 13. Qingxi daoren 1986: 182-188.

29. Chan zhen yishi chapitre 5. Qingxi daoren $1986: 65$.

30. Voir ci-dessus note 21 .

31. Chan zhen yishi chapitre 6. Qingxi daoren $1986: 70-71$. L'explication du poème, qui, grâce à une suite de jeux de mots permet d'identifier les composants des caractères chinois qui forment le nom de la belle, défie quelque peu la traduction... On me permettra d'en donner ici en version originale le début (qui ne livre la clef que du nom de famille, Li 黎), à l'intention du lecteur sinisant curieux : 他夢中對你道：“田中 有稻側半初, 人下小小是阿奴。' 這兩句是拆白的話, 講出他那姓來。田中 
la profession d'entremetteuse, elle s'occupera bien vite d'arranger un rendezvous galant entre le moine et la belle. Mais quand le moine se trouve de nouveau en position de passer à l'action, à l'état de veille cette fois, il en est de nouveau empêché, car on découvre que Li Saiyu a ses règles. Zhong Shoujing, entré dans les ordres encore enfant, est d'une telle ignorance des femmes qu'il ne sait même pas de quoi il s'agit ! Lorsque Li Saiyu lui prodigue les explications nécessaires, il comprend néanmoins aussitôt la signification de la dernière partie de son rêve : le dhūta au visage écarlate surgissant d'une fente de la muraille pour prévenir leurs ébats annonçait les menstrues contrariantes de Li Saiyu ${ }^{32}$. Il faudra attendre une troisième rencontre pour que les amants puissent enfin assouvir leur désir.

\section{Conséquences à long terme du songe : effets de miroir et articulations narratives}

Au-delà de leurs effets immédiats (interprétations justes et erronées, compréhension ultérieure des présages), ces deux épisodes oniriques ont des échos diégétiques plus lointains, en se voyant reflétés par plusieurs moments ultérieurs du récit ou en fonctionnant comme des points d'articulation de celuici.

Examinons d'abord dans cette optique le songe de Zhong Shoujing. Son rêve érotique interrompu n'a pas mis un terme à son obsession mais l'a laissé triste et abattu. Il voit s'éloigner de lui l'état de sainteté qu'il croyait sincèrement poursuivre au début du récit. Sa quête d'explication provoque l'entrée dans le récit de Zhao «Bouche-de-miel », autour de laquelle graviteront tout un noyau de personnages négatifs, et qui aidera le jeune et naïf Zhong Shoujing à se métamorphoser en moine hypocrite prêt à tout pour arriver à ses fins et à masquer ses débauches. Au chapitre 8 , Zhong, retenant les leçons de « Bouche-de-miel » sur l'efficacité des présages vus en songe, n'hésitera pas en effet à inventer cyniquement de toutes pièces une prétendue rencontre divine - qu'il dit avoir obtenue dans la méditation (chanding), mais qui est aussi bien qualifiée de vision faite « en rêve » mengli, ou encore de "présage onirique » (mengzhao) - pour convaincre un haut fonctionnaire de céder au monastère un bâtiment dont il entend faire le refuge de ses amours illicites ${ }^{33}$.

有稻是禾字, 側半初是側邊加半個初字, 人下小小是尔字, 湊完成卻不是個黎 字? 他與你講道他姓黎。

32. Chan zhen yishi chapitre 7. Qingxi daoren 1986: 87.

33. Chan zhen yishi, chapitre 8. Qingxi daoren 1986 : 103-104. 
La transformation de Zhong Shoujing en mauvais moine permet en parallèle au romancier d'achever de camper le personnage de Lin Danran comme héros positif central de l'histoire : moine lui-aussi, cet ancien officier des Wei ayant pris la tonsure a été envoyé sur ordre impérial aux côtés du trop délicat et civil (wen) Zhong Shoujing comme abbé militaire ( $w u$ ) adjoint chargé de la sécurité du monastère. Ayant essayé en vain de mettre en garde son collègue, Lin ne fait que susciter son hostilité, et, calomnié par lui auprès de l'empereur Wu des Liang, doit prendre la fuite, entamant ainsi la longue errance qui lui permettra de rassembler autour de lui valeureux disciples et fidèles compagnons : elle constituera l'essentiel du récit. ${ }^{34}$

Le rêve de Chen Abao trouve un écho encore plus caractéristique dans une autre part du récit : cet écho intervient au chapitre 20, donc juste au milieu du roman. On y voit un personnage qui rappelle en bien des points Abao effectuer un voyage aux enfers : comme Abao, il est à ce moment du récit connu sous un sobriquet familier, quoique sensiblement plus péjoratif, Achou (« le mocheton»). Comme lui, il est indocile, jeune et pauvre (orphelin ignorant tout de ses origines, il vit de la charité de ceux qui l'ont recueilli) - et surtout, tout comme Abao, il va, à la suite d'une rencontre avec les autorités de l'autre monde, avoir révélation de ses origines. Le goût des auteurs de tongsu xiaoshuo de cette époque pour les effets de répétition et d'écho était trop constant pour qu'on puisse n'y voir qu'un simple hasard ${ }^{35}$. Mais si le personnage d'Achou peut être considéré comme reproduisant comme par un jeu de miroir celui d'Abao, il en est le reflet inversé : ce n'est pas face à un juge sévère qu'il se retrouve en enfer, comme Abao, mais devant un personnage bienveillant, son propre père, devenu après son trépas magistrat infernal. Le jeune orphelin apprendra ainsi qu'il n'est autre que le fils de Du Chengzhi, celui-là même dont la mort avait été causée par la délation d'Abao. Comme Achou se trouve, suite à un concours de circonstances, avoir été recueilli par Lin Danran, lequel doit d'avoir eu la vie sauve au sacrifice de Du Chengzhi, son statut se verra spectaculairement réévalué à son retour auprès du moine guerrier : fils du sauveur de celui qui l'a recueilli, il retrouve honorabilité ainsi

34. De manière significative, les seuls rêves qui sont prêtés à Lin Danran sont des rêves yin au message sans ambiguïté : au chapitre 20 il se voit demander de venir en aide à un moine défunt châtié pour des fautes non réparées ; lorsqu'il a pu apurer la dette du mort, ce dernier lui apparaît de nouveau en songe pour le remercier et lui annoncer qu'il est désormais autorisé à se réincarner.

35. C'était ce que le grand commentateur Jin Shengtan (1608-1661) désignait dans son commentaire à Au bord de l'eau par le terme de Zhengfan fa «L'offense caractérisée » et Lüefan fa « l'offense légère », à savoir « le tour de force consistant à répéter un thème en en variant les péripéties » : Dars 1978 : cxxviii. 
que son véritable nom, Du Fuwei ${ }^{36}:$ il deviendra le principal protagoniste de la partie suivante du roman.

Il importe de relever que le périple infernal de Achou/Du Fuwei n'est pas un rêve, car Achou est mort pour de bon au début de l'épisode : il s'est rompu le cou en tombant d'un arbre. Ainsi, quand il est question de le renvoyer dans le monde des vivants, on s'inquiète de ce que son corps ait déjà commencé à se décomposer : il convient de hâter son retour ${ }^{37}$. Comme on peut observer par ailleurs que les courts voyages aux enfers que l'on rencontre fréquemment dans le roman en langue vulgaire sont le plus souvent désignés comme des rêves faits par le voyageur ${ }^{38}$, il convient de se demander si l'auteur de Chan zhen yishi n'a pas cherché en insistant sur la mort réelle bien que réversible d'Achou à marquer la différence avec le songe d'Abao, qu'il réplique en partie : l'expérience d'Achou, plus qu'une simple révélation de ses origines, le fait passer par un véritable processus de mort et de renaissance, et imprimera à l'existence du personnage comme au cours du roman un tournant décisif. La mise en garde nébuleuse reçue en songe par Abao restera quant à elle incomprise et n'altérera pas son destin : ici se distinguent peut-être l'espace onirique ambigu et trompeur, et la réalité héroïque, simple et vraie.

Chan zhen yishi comprend une troisième séquence onirique complexe que nous n'avons pas encore évoquée. Elle est sensiblement différente des deux rêves précédent du fait que le rêveur y est un personnage positif : on aura peut-être remarqué que, jusqu'ici, tout se passait comme si un rêve complexe ne pouvait être prêté qu'à un personnage à la moralité douteuse ${ }^{39}$. Le héros est ici Zhang Shanxiang : il est, parmi les trois disciples de Lin Danran, celui qui incarne le talent lettré par opposition aux vertus martiales incarnées par Xue Ju et Du Fuwei. Mais si le rêve qu'il fait au chapitre 31 n'est pas à proprement parler un cauchemar, il surgit, comme les deux autres songes, du désir et de l'inquiétude mêlée. Alors que ses deux frères jurés sont partis guerroyer, Zhang Shanxiang, resté auprès du maître, tue accidentellement un bon à rien, ivrogne et violent, que son cheval a piétiné. Obligé de prendre la fuite, il se réfugie dans le jardin d'une riche demeure où il surprend la conversation d'une très belle jeune fille et de ses suivantes. Resté seul dans le jardin, il y découvre

36. Chan zhen yishi chapitre 20. Qingxi daoren 1986:296-300.

37. Chan zhen yishi chapitre 20. Qingxi daoren,1986:300.

38. Je me permettrais de renvoyer ici à mon mémoire d'habilitation à diriger des recherches, «La Descente aux enfers »: Durand-Dastès 2011 : 62-84.

39. On peut songer ici à la remarque de Cheng Yi 程頣 (1033-1107) : « Si le saint n'a pas de rêves, c'est que son énergie vitale est pure, mais le sot rêve beaucoup parce que son énergie vitale est trouble ». Cité et traduit dans Diény 2001 : 133-134. 
un mouchoir sur lequel la jeune fille a inscrit un poème non seulement fort gracieusement composé, mais qui comporte des implications sensuelles tout à fait prometteuses. Ne voulant révéler prématurément sa présence, il se réfugie dans la chapelle d'une divinité située au milieu du jardin où il s'endort : apparaissant dans son songe, le dieu lui révèle son propre nom : il était de son vivant le général de la période des Trois royaumes, Ma Teng. Surtout, la divinité révèle au jeune homme le nom de la belle, Duan Linying, qui n'est autre que la fille de Duan Shao, grand officier du pays de Qi, et accepte de célébrer sur le champ son mariage avec elle. La belle paraît aussitôt, la noce est organisée, et Zhang Shanxiang est aux anges, lorsque le rêve printanier prend fin en tournant brièvement au cauchemar : le rituel nuptial est interrompu par l'irruption des sbires du yamen venus se saisir du meurtrier de l'ivrogne. Zhang se réveille en sursaut, couvert de sueur. Il se rendort bientôt et ne rêve plus le reste de la nuit ${ }^{40}$.

Le lendemain, découvert par les servantes, il s'arrange pour être autorisé à demeurer dans le jardin quelques jours. Il flirte avec l'une d'entre elles, la peu farouche Chunxiang, qui devient la protagoniste d'un second rêve, très bref : alors qu'elle a promis de rejoindre Zhang pendant la nuit, celui-ci s'endort et rêve que la fille arrive et se déshabille devant lui...mais il se réveille alors en sursaut. À peine un instant plus tard, Chunxiang arrive et couche pour de bon avec lui : ici, le désir exprimé par le rêve a trouvé sans aucun délai son assouvissement ${ }^{41}$. Chunxiang, gagnée par l'étreinte avec Zhang à la cause de celui-ci (elle se promet d'être concubine dans l'union matrimoniale projetée), joue dans les pages suivantes le rôle classique d'entremetteuse dévolu aux soubrettes dans le théâtre et le roman en langue vulgaire : la belle Linying a tôt fait de promettre le mariage à Zhang et échange avec lui serments et gages de fidélité avant que le jeune homme ne soit finalement mis à la porte par la mère de la jeune fille. Il s'en va, mais son rêve autant que son succès à plaire à sa promise le convainquent que ses projets matrimoniaux seront couronnés de succès - ce qui se réalisera en effet dans les derniers chapitres du roman.

Comme le rêve de Zhong Shoujing, premier mouvement de la séquence des débauches au monastère Miaoxiang, est l'acte déclencheur de la carrière de Lin Danran parmi les braves « des rivières et des lacs » (chapitres 5 à 20), comme le motif du double périple infernal d'un jeune gueux inaugure la séquence des chapitres 20 à 30 où Du Fuwei, ayant retrouvé sa noble extraction, conduit le récit par ses exploits hérö̈ques, le songe de Zhang Shanxiang ouvre en effet la dernière partie du roman (chapitres 31 à 40). Elle verra, grâce à l'alliance

40. Chan zhen yishi, chapitre 32. Qingxi daoren $1986: 488-490$.

41. Chan zhen yishi, chapitre 32. Qingxi daoren 1986: 497. 
matrimoniale nouée par lui avec le puissant clan des Shao, les trois frères jurés se rassembler un moment sous la bannière des Qi avant de participer ensemble à la fondation de la dynastie des Sui.

Rêves printaniers ou périples infernaux interviennent ainsi à des moments clefs du roman et, chacun à leur façon, annoncent ses développements futurs. Le rêve est ici dans son rôle premier, celui d'un récit enchâssé dans le "grand récit » de la vie, qu'il permet d'infléchir ou d'expliquer. Il n'est donc pas étonnant de voir rappelé sa fonction prédictive. Pourtant, comme on va le voir, un roman en langue vulgaire tel que Chan zhen yishi sait jouer de la dimension oniromantique tout en s'en affranchissant partiellement.

\section{Lieux communs oniromantiques et traits idiosyncratiques des rêves de Chan zhen yishi}

Les rêves que nous avons présentés puisent chacun à des topoï bien connus, qui n'appartiennent pas en propre au roman en langue vulgaire. Ainsi la vision onirique d'images énigmatiques, souvent présentées sous la forme des rébus graphiques du chaizi, et dont le décryptage permet de prévoir un événement ou d'éclairer sur la destinée, est un lieu commun des traités divinatoires.

Quant aux descentes provisoires aux enfers qui éclairent un personnage sur son destin ou celui de ses proches, et servent souvent de mise en garde, elles se rencontrent dans les anecdotes prodigieuses ou la littérature didactique depuis au moins les Six dynasties ${ }^{42}$.

Le rêve de Zhang Shanxiang, enfin, rappelle fortement un topos divinatoire bien connu, celui du rêve incubatoire : un fidèle s'endort dans le temple d'une divinité en espérant que celle-ci lui envoie un rêve destiné à éclairer son destin. Au chapitre 36, on dira ainsi que ce rêve « a été offert par le Grand Prince Ma Teng » (Ma Teng da wang ci meng $)^{43}$ : on ne parlerait pas autrement d'un songe révélateur octroyé par le dieu sollicité par le rêveur ${ }^{44}$.

L'originalité de nos récits consiste à mêler ces lieux communs divinatoires à des inventions tout à fait originales, où destinée et hasard se voient habilement mêlées. Le songe de Zhang Shanxiang, tout en rappelant la procédure par laquelle un rêveur sollicite une révélation onirique, intervient sans aucune

42. Sur le motif médiéval du voyage aux enfers et son évolution, voir Campany 1990, 1995 ; Wang Li 2003.

43. Chan zhen yishi, chapitre 36. Qingxi daoren $1986: 562$.

44. Sur le rêve incubatoire, voir la belle étude de Brigitte Baptandier sur un sanctuaire du Fujian dont c'est une des activités principales : Baptandier 1996. 
intention de la part du rêveur : il ignore jusqu'à l'identité du dieu, et ne se trouve dans sa chapelle que parce qu'elle lui offre la meilleure cachette. Il y apprendra pourtant tout à la fois le nom de la divinité et celui de la belle, et se verra promis le mariage avec cette dernière sans avoir consciemment espéré cette annonce heureuse, bien que n'ayant formulé nulle prière : celle de son désir latent a suffi au dieu entremetteur.

Le rêve de Chen Abao, s'il suit étroitement le scénario «classique » d'un jugement aux enfers (arrestation du «prévenu » par des sbires démoniaques qui le défèrent devant un magistrat dont les pouvoirs permettent de consulter les archives des existences présentes comme des vies antérieures), est rendu plus vivant et intrigant par ce qu'on ne peut appeler autrement qu'une fantaisie religieuse : la rencontre n'a en effet pas lieu au cours d'un périple catabatique, mais à la surface d'une rivière. Bien plus, le personnage qui agit comme un roimagistrat du monde des ténèbres n'est pas Yama ou un autre des Dix rois des enfers, mais un énigmatique « Roi de lumière de la loi orthodoxe des préfectures aquatiques » (Shuifu zhengfa mingwang) ${ }^{45}$. Aucune divinité portant ce nom n'est inscrite à ma connaissance dans la longue liste du panthéon chinois. Il est plus que vraisemblable que notre auteur s'inspire ici de la mythologie de l'antique figure de «l'officier des eaux », Shuiguan, un des «trois officiers » (sanguan) : ainsi que l'a rappelé Michel Strickman, dans les croyances taoïstes anciennes ces divinités avaient juridiction sur les défunts, et « l'officier de l'eau » incarnait la dimension la plus punitive de leur autorité :

« Dans le premier système taoïste, il y avait trois juges des morts : les officiers du ciel, de la terre et de l'eau. Le membre le plus redouté de ce trio était l'officier de l'eau, et la pire destinée qui pouvait attendre les âmes des morts était d'être voués à l'office de l'eau (shuifu 水府). L'officier de l'eau avait, de toute évidence, hérité de la substance et de la fonction infernale de l'ancien comte de l'eau $(\mathrm{Hebo})^{46}$. »

Dans la vision d'Abao toutefois, l'antique figure de l'officier des eaux se voit mâtinée de traits empruntés à une figure divine plus récemment apparu dans le panthéon chinois : celle des divinités courroucées venues du bouddhisme ésotérique, les Mingwang « Rois de lumière » ou « Rois de science » : les peintures et statues de ces effrayants personnages, aux multiples têtes munies de crocs et bras brandissant des armes, impressionnèrent tant les fidèles chinois qu'on les rencontre en bien des secteurs de la culture savante

45. Chan zhen yishi, chapitre 12. Qingxi daoren $1986: 178$.

46. Strickmann $1996: 399$. 
et populaire, bien au-delà du bouddhisme proprement dit ${ }^{47}$. Bref, le romancier de Chan zhen yishi semble avoir ici façonné librement, grâce à des éléments préexistants, un magistrat infernal n'appartenant qu'à son imagination. Par ailleurs, si l'on considère l'ensemble de la séquence onirique qui se clôt par la rencontre avec le juge de l'autre monde, on remarque qu'elle est précédée par ce qu'on pourrait appeler un « pur » cauchemar, qui ne contient quant à lui aucun élément prophétique et ne puise donc pas aux sources de la littérature religieuse ou divinatoire préexistante. C'est une fois le tumulte émotionnel qui agite l'esprit du scélérat purgé par un premier rêve que le récit lui fera affronter le songe annonciateur de son châtiment. Un peu de la même façon, le rêve qui annonce son destin matrimonial à Zhang Shanxiang est suivi d'un autre, non prophétique, où son désir fait simplement anticiper par Zhang l'arrivée de la soubrette déjà en chemin pour se donner à lui.

On retrouve la même combinaison du vocabulaire divinatoire banal et de la libre invention romanesque dans le rêve érotique de Zhong Shoujing : l'irruption du « dhūta au visage écarlate » surgi d'une fente de la muraille ne peut en aucun cas, à la différence des couplets énigmatiques récités par $\mathrm{Li}$ Saiyu, être décryptée par la science divinatoire de la roublarde «Bouche-demiel » : cette image frappante, annonçant au sein d'un rêve issu d'un désir frustré une nouvelle frustration à venir est proprement incompréhensible en tant qu'énoncé divinatoire, mais laisse le lecteur soupçonner un rebondissement narratif à venir dont il ne peut encore deviner la teneur.

\section{De la logique mantique à la psychologie ?}

Ces rêves n'apparaissent pas n'importe où dans le récit, car ils prennent place à des moments d'intense frustration, où les personnages sont poursuivis par leurs fautes ou ébranlés par leurs désirs inassouvis. Si le romancier de Chan zhen yishi leur fait en quelque sorte traverser tous les lieux-communs oniromantiques, il y dispose aussi pour eux traits particuliers ou situations résolument originales. Ce faisant, il semble se libérer progressivement du vocabulaire divinatoire dans la construction de ses séquences oniriques. Nous illustrerons cette démarche par un rêve emprunté non à Chan zhen yishi, mais à sa suite Chan zhen houshi, écrite quelques années plus tard. La protagoniste, Lao Woxi, est l'une des quatre jeunes concubines d'un vieillard impuissant,

47. Sur cette question je me permettrai de renvoyer à mon étude récente sur le bouddhisme tantrique vu par le roman en langue vulgaire des Ming et des Qing : Durand-Dastès 2016. 
de surcroît absent de chez lui à ce moment du récit. Un soir, elle surprend les échos d'une discussion galante entre une autre des concubines et un moine qui, sous prétexte de récitation bénéfiques de sūtra, a été imprudemment introduit par l'épouse principale dans la maison. Sans en croire tout à fait ses oreilles mais profondément troublée de ce qu'elle a surpris, elle regagne sa chambre dans un état d'excitation extrême. Elle plonge bientôt dans un sommeil agité où elle fait un songe saturé de symbolisme sexuel : elle y rêve d'un moine qui bat rythmiquement un tambour à l'aide d'un bâton, de la crue subite d'une rivière, de son corps qui s'enflamme soudain et doit être aspergé par le moine d'eau glacée, pour littéralement «éteindre son feu ${ }^{48} » .$. . Mais aucun de ces traits significatifs n'est commenté ultérieurement, que ce soit par un autre personnage ou par le narrateur, et le seul présage que contient le rêve est celui de la nuit d'amour qu'elle passera bientôt avec le moine, prémisse du déchaînement érotique qui emportera sous peu toute la maisonnée. Tout au plus son rêve la distingue-t-elle parmi les concubines comme celle dont le désir est à la fois le plus fort et le mieux assumé : elle vantera les talents amoureux du moine à une troisième concubine, Tian, afin de la convaincre de rejoindre le groupe adultère, et, à l'issue de cet épisode pornographique et funèbre, elle restera la seule survivante parmi les quatre concubines ${ }^{49}$...Le rêve printanier de Lao Woxi n'est donc pas sans importance narrative, mais ses composants ne trouvent pas d'écho individuellement identifiables dans la suite du récit. On pourrait être tenté de conclure que, en délaissant progressivement les éléments conventionnels (mots et images énigmatiques à décrypter, récits types) transmis par la tradition mantique, Fang Ruhao participe à la création d'un langage de psychologie onirique « moderne » prêt à être réemployé par les romanciers des générations suivantes, au premier rang desquels l'auteur $\mathrm{du}$ « Rêve dans le Pavillon rouge ». On se gardera toutefois d'une vision trop téléologique, qui conduirait le roman chinois d'un stade « religieux » archaïque vers une « modernité » psychologisante. Car l'art d'un Fang Ruhao réside peut-être justement dans sa capacité à savoir aussi bien ré-agencer les données des clefs des songes et du discours mantique de son temps que prêter à ses

48. Chan zhen houshi, chapitre 51. Fang Ruhao 1993: 460. Keith McMahon traduit intégralement ce rêve : McMahon 1988 : 121-122.

49. Le moine, doté d'une puissance sexuelle quasi monstrueuse, fera malgré lui périr dans ses bras la quatrième concubine. Le scandale ayant éclaté, les deux autres mourront peu après de honte et de choc mêlé. Seule Woxi survivra, pour être revendue par l'épouse principale, pressée d'effacer la dernière trace de l'affaire. Les chapitres narrant ce conte érotique noir sont ceux qui sont expurgés dans la version en cinquante-trois chapitres du roman. 
Les usages narratifs du rêve dans un roman fleuve chinois du XVII ${ }^{\mathrm{e}}$ siècle

rêveurs des traits inexpliqués issus tout droit de son imagination d'écrivain... ou de ses propres désirs et songes.

\section{BIBLIOGRAPHIE}

BAPTANDIER Brigitte (1996). « Entrer en montagne pour y rêver : le mont des Pierres et des Bambous ». Terrain, $26: 99-122$. En ligne [http://terrain.revues.org/ index3160.html\#txt].

BAUER Wolfgang (1979). «Chinese Glyphomancy (ch'ai tzu) and its Uses in Present Day Taiwan ». In Allan S., and COHEN A. P. (dir.), Legend, Lore and Religion in China. Essays in Honor of Wolfram Eberhard on his Seventeenth Birthday. San Francisco (CA) : Chinese Materials Center.

CAMPANY Robert Ford (1990). «Return-from-Death Narratives in Early Medieval China ». Journal of Chinese Religions, 18 : 91-125.

CAMPANY Robert Ford (1995). « To Hell and Back : Death, Near-Death, and OtherWorldly Journeys in Early Medieval China ». In Collins and FishBANE, Death, Ecstasy, and Other Worldly-Journeys. New York, SUNY Press : 343-360.

CHAN ZHEN HOUSHI 禪真後史 [https://zh.wikisource.org/wiki/禪真後史] [consulté le 16 décembre 2017].

CHAN ZHEN YISHI 禪真逸史 [http://ctext.org/wiki.pl?if=gb\&res=542916\&remap=gb] [consulté le 16 décembre 2017].

CHAN ZHEN YISHI 禪真逸史 [http://open-lit.com/bookindex.php?gbid=16] [consulté le 16 décembre 2017].

CHEN Shiyuan (2008). Wandering Spirits : Chen Shiyuan Encyclopedia's of Deams, transl. with an introduction by Richard E. STRASSBERG. Berkeley, University of California Press.

DARS Jacques (1978). «Introduction », Au bord de l'eau. Paris, Gallimard (« Bibliothèque de la Pléiade »).

DIÉNY Jean-Pierre, (2001). «Le saint ne rêve pas : de Zhuangzi à Michel Jouvet ». Études chinoises, XX(1-2) : 127-200.

DoNG Yue 董說 (1983). Xiyou bu 西遊補. Shanghai, Shanghai guji.

DrETTAS, Dimitri (2007). Le Rêve mis en ordre. Les traités onirologiques des Ming à l'épreuve des traditions divinatoire, médicale et religieuse du rêve en Chine, thèse de doctorat. Paris, EPHE.

DURAND-DASTÈS Vincent (2016). «Rencontres hérétiques dans les monastères de Kaifeng : le bouddhisme tantrique vu par le roman en langue vulgaire des Ming et des Qing ». In DURAND-DASTÈS Vincent (dir.), Empreintes du tantrisme en Chine et en Asie orientale : imaginaires, rituels, influences. Louvain, Peeters (« Mélanges chinois et bouddhiques »,32) : 27-62.

DURAND-DASTÈS Vincent (2011). «Littérature narrative et religions chinoises du XII au XIX ${ }^{\mathrm{e}}$ siècle : un surnaturel bien de ce monde. Synthèse de l'activité scientifique 
(2000-2010) en vue de l'obtention de l'habilitation à diriger des recherches ». Paris : Inalco. En ligne [https://tel.archives-ouvertes.fr/tel-01069129/document] consulté le 16 décembre 2017.

DURAND-DASTÈS Vincent (2008). La Conversion de l'Orient : un périple didactique de Bodhidharma dans un roman chinois en langue vulgaire du XVII siècle. Bruxelles : Institut Belge des Hautes Études Chinoises (« Mélanges chinois et bouddhiques », 29).

DURAND-DASTÈs Vincent (2007). «Poisons exotiques et vices domestiques : de vertueux héros aux prises avec les $g u$ 劃 dans un roman du XVII ${ }^{\mathrm{e}}$ siècle ». Études chinoises, vol. XXVI : 83-106.

FANG Ruhao 方汝浩 (1993). 《Chan zhen houshi 禪真後史 》, édité par Wang Yimin 王逸民. In Hou Zhongyi 侯忠義 (dir.), Mingdai xiaoshuo jikan 明代小說輯刊, 4(1). Chengdu, Ba Shu shushe.

Gollut Jean-Daniel (1993). Conter les rêves : la narration de l'expérience onirique dans les æeuvres de la modernité. Paris, José Corti.

Hegel Robert E. (1981). The Novel in Seventeenth-Century China. New York, Columbia University Press.

JIA Haijian 賈海建 (2013). «Mingdai xiaoshuo jia Qingxi daoren kaobian 明代小說家 清溪道人考辨》. Ming Qing xiaoshuo yanjiu 明清小說研究, 108(2) : 199-209.

LACKNER Michael (1985). Der chinesische Traumwald. Traditionelle Theorien des Traumes und seiner Deutung im Spiegel der ming-zeitlichen Anthologie Meng-lin hsüan-chieh. Frankfurt/Bern/New York, Peter Lang.

LÉVY André (trad.) (1998). Histoire extraordinaires et récits fantastiques de la Chine ancienne : chefs-d'œuvre de la nouvelle (Dynastie des Tang, 618-907), II. Paris, GF-Flammarion.

LIU Wenying 劉文英, CAO Tianyu 曹田玉 (2003). Meng yu Zhongguo wenhua 夢與中 國文化. Pékin, Renmin chubanshe.

McMahon, Keith (1988). Causality and Containment in Seventeenth Century Chinese Fiction. Leyde, Brill.

ŌTsUKA Hidetaka 大塚秀高 (1987). Zōho chūgoku tsūzoku shōsetsu shomoku 增補中 國通俗小説書目 (Bibliographie du roman chinois en langue vulgaire, augmentée et complétée). Tokyo, Kyukoshoin.

QINGXI daoren 清溪道人 (1986). Chan zhen yishi 禪真逸史. Haerbin, Heilongjiang renmin.

QINGXI daoren 清溪道人 (1990). Chan zhen yishi 禪真逸史. Shanghai, Shanghai guji chubanshe (3 vol.).

SHI Lei 石雷 (2004). Notices “Chan zhen yi shi” et “Chan zhen hou shi”. In SHI Changyu 石昌渝, LI Jianguo 李劍國 et al. (dir.), Zhongguo gudai xiaoshuo zongmu : baihua juan 中國古代小說總目: 白話卷. Taiyuan, Shanxi jiaoyu chubanshe : 25-26.

SONG Yan 宋艷 (2014). «Chan zhen yishi chengshu shijian kaozheng 禅真逸史成書 時間考證》. Anhui wenxue, 370(5) : 9-11. 
STRASSBERG Richard (2014). «Glyphomantic Dream Anecdotes ». In CHEN Jack et David SCHABERG (dir.), Idle Talk : Gossip and Anecdote in Traditional China. Berkeley, University of California Press.

STRASSBERG Richard E. (2008). "Introduction”. In CHEN Shiyuan, Wandering Spirits : Chen Shiyuan Encyclopedia's of Dreams. Berkeley, University of California press.

STRICKMANN Michel (1996). Mantras et mandarins : le bouddhisme tantrique en Chine. Paris, Gallimard.

STRUVE, Lynn A. (2007). « Dreaming and Self-Search during the Ming Collapse : The Xue Xiemeng biji, 1642-1646 ». T'oung pao, 96(3) : 159-192.

TARUMOTO Teruo 樽本照雄 (2002). Zengbu xinbian Qing mo Min chu xiaoshuo mulu 清末明初小説目錄 (《Bibliographie des romans de la fin des Qing et du début de la période républicaine, nouvellement publiée et augmentée »). Ji'nan, Qi Lu shushe 齊魯書社.

VANCE Birgid (2017). «Deciphering Dreams : How Glyphomancy Worked in Late Ming Dream Encyclopedic Divination ». The Chinese Historical Review, 24(1) : 5-20.

VANCE Birgid (2012). « Textualizing Dreams in a Late Ming Dream Encyclopedia », Ph. D. Princeton, Princeton University.

WAN Qingchuan 万晴川 (2005). «Meng you ji xiong : gudai xiaoshuo yu zhan meng 夢 有吉凶古代小説與占夢》. In ID., Zhongguo gudai xiaoshuo yu fangshu wenhua 中國古代小説與方術文化. Beijing, Zhongguo shehui kexue chubanshe : 222237.

Wang Li 王立 (2003). «Zhongguo gudai mingyou muti jizhong leixing ji yanbian guocheng 中國古代冥游母體幾種類型及演變過程 ». Dongnan daxue xuebao 東南大學學報, 3 .

WANG Qingyuan 王清原, Mou Renlong 牟仁隆, HAN Xiduo 韓錫鐸 (2002). Xiaoshuo shufang lu 小説書坊錄 (Registre des imprimeurs de romans). Beijjing, Beijing tushuguan chubanshe.

ZEITLIN Judith (1993). Historian of the Strange : Pu Songling and the Chinese Classical Tale. Stanford, Stanford University Press. 
Vincent Durand-Dastès

\section{GLOSSAIRE}

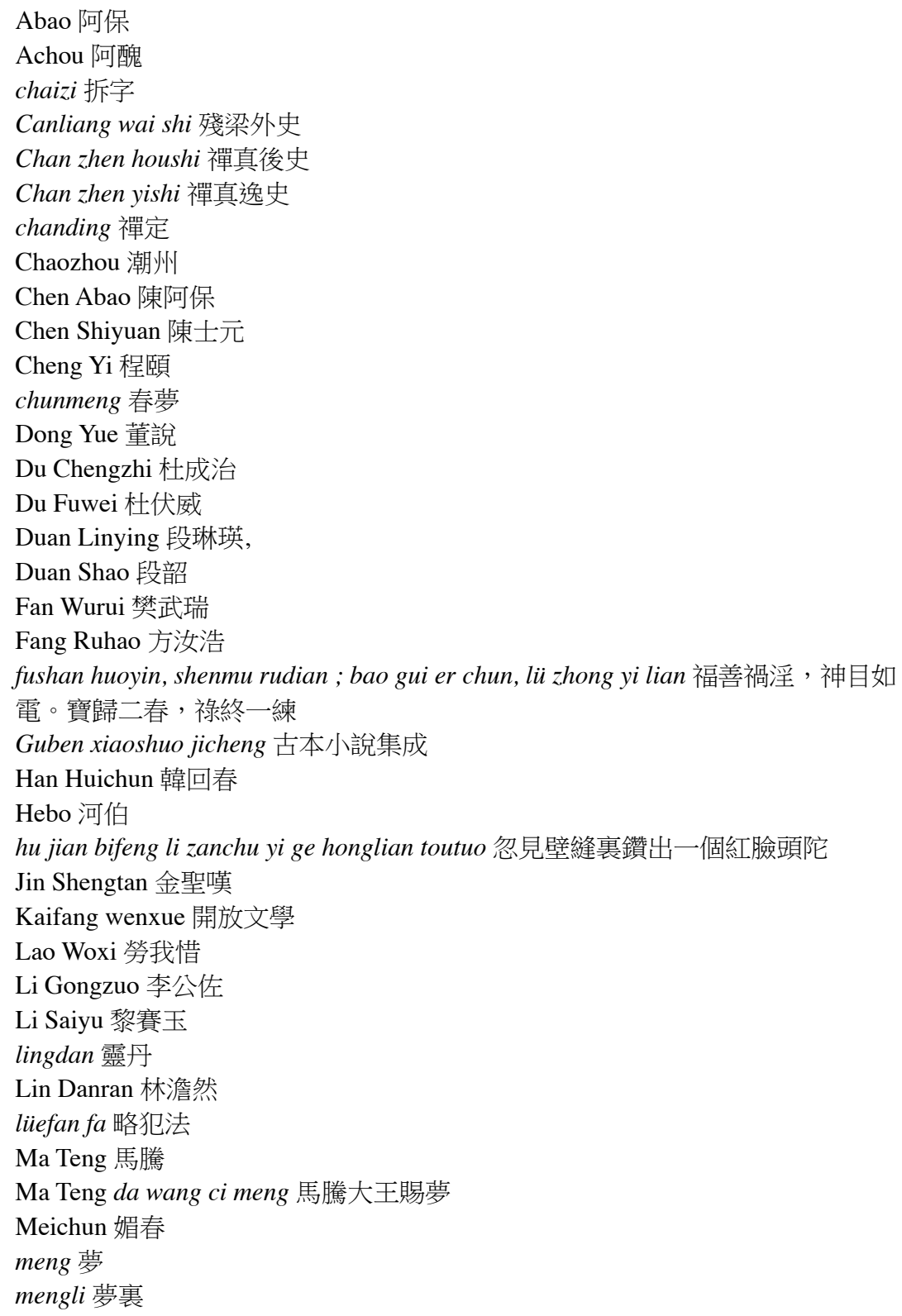


Les usages narratifs du rêve dans un roman fleuve chinois du XVII siècle

menglong 朦朧

Mengzhan yizhi 夢占逸旨

mengzhao 夢兆

Miaolong 妙龍

Miaoxiang si quanzhuan 妙相寺全傳

Mingdai xiaoshuo jikan 明代小說輯刊

mingwang 明王

Nanke taishou zhuan 南柯太守傳

Nanke yi meng 南柯一夢

Qi 齊國

Qingxi daoren 清溪道人

sanguan 三官

Shen Quan 沈全

Shuiguan 水官

Shuifu 水府

Shuifu zhengfa mingwang 水府正法明王

tongsu xiaoshuo 通俗小說

toutuo 頭陀

wen 文

$w u$ 武

xing 醒

Xiyou bu 西遊補

Xue Ju 薛舉

yan 魘

yao 爻

yinmeng 陰夢

Yongqing 永清長老

Zhang Shanxiang 張善相

Zhao mizui 趙蜜嘴

zhengfan $f a$ 正犯法

Zhong Shoujing 鐘守淨 
